FERMILAB-TM-2555-APC

Beams-doc- 4328

\title{
Theory and Simulations on $g-2$ Proton Beam Preparation
}

\author{
K.Y. Ng \\ Fermi National Accelerator Laboratory, P.O. Box 500, IL 60510
}

April, 2013

\begin{abstract}
A Booster batch consisting of $8453-\mathrm{MHz}$ bunches needs to be debunched and recaptured into four $2.5-\mathrm{MHz}$ super bunches, to be extracted to hit a target to generate muons for the $g-2$ experiment. These super bunches must be each of full width $\leq 120$ ns. In addition, the whole beam preparation procedure as well as eventual bunch-by-bunch extraction must be performed within two Booster cycles or $133 \mathrm{~ms}$. Machine studies are required in the Main Injector to demonstrate the feasibility of the preparation. The theory, including adiabatic debunching, adiabatic re-capture, and some other relevant issues necessary for the machine studies, is discussed.
\end{abstract}




\section{Introduction}

The proton source of the $g-2$ experiment at Fermilab consists of first injecting a booster batch of 84 bunches into the Recycler Ring. The Booster batch is of length $\sim 1.6 \mu \mathrm{s}$. The batch is debunched adiabatically using $53-\mathrm{MHz}$ rf. After that the beam is re-bunched into four narrow super-bunches using $2.5-\mathrm{MHz}$ rf. It is critical that each of these super bunches be of full width $\lesssim 120$ ns. Otherwise the muon beam generated later will be too long to fit into the $g-2$ ring. Moreover, the time duration of the above rf maneuvering as well as eventual bunch-by bunch extraction is limited, all procedures must be finished within two Booster cycles, or $133 \mathrm{~ms}$. For this reason, machine studies are necessary to demonstrate the feasibility of the above rf maneuvering. Since $53-\mathrm{MHz}$ and $2.5-\mathrm{MHz}$ rfs were not available in the Recycler Ring before the 2012 Fermilab shutdown, the studies had to be performed in the Main Injector instead. This article outlines the theory behind the machine studies.

In Sect. 2, we first describe the ideal situation of adiabatic debunching and adiabatic capture when there is no time limitation. Realistic adiabatic debunching is studied in Sec. 3 and realistic adiabatic capture is studied in Sec. 4. In Sec. 5, we estimate the voltage upgrade of the 2.5-MHz rf to be moved from the Main Injector to the Recycler Ring. In Sec. 6, we study the problem of particle leakage from both sides of the Booster batch during adiabatic debunching and adiabatic capture. We compute the required rf barrier strength required to stop particle leakage at both end of the Booster batch during adiabatic debunching and re-capture. Finally in Sec. 7, the conclusion is given.

\section{Ideal Adiabatic Debunching and Adiabatic Capture}

The Booster bunches are often quoted to have a $95 \%$ area of $0.1 \mathrm{eVs}$ each in the $53-\mathrm{MHz}$ rf buckets. Twenty one of these bunches are merged into a super bunch using $2.5-\mathrm{MHz}$ rf. Ideal adiabatic debunching of these 21 Booster bunches will lead to a flat distribution having bunch area $A=2.1 \mathrm{eVs}$. Subsequent ideal adiabatic capture into the $2.5-\mathrm{MHz}$ bucket at $V=54.26 \mathrm{kV}$ results in a super bunch of half width $\hat{\tau}=50.0$ ns and energy spread $\widehat{\Delta E}=13.46 \mathrm{MeV}$. We have used the formulas:

$$
\widehat{\Delta E}=\sqrt{\frac{2 e V \beta^{2} E_{0}}{\pi h|\eta|}} \sin \frac{h \omega_{0} \hat{\tau}}{2},
$$




$$
A=\sqrt{\frac{128 e V \beta^{2} E_{0}}{\pi h^{3} \omega_{0}^{2}|\eta|}}[E(m)-(1-m) K(m)],
$$

where $K(m)$ and $E(m)$ are complete elliptic functions of the first and second kind with argument $m=\sin ^{2}\left(h \omega_{0} \hat{\tau} / 2\right), h=28$ is the $2.5-\mathrm{MHz}$ rf harmonic of the Main Injector, $\omega_{0} / 2 \pi=89.82 \mathrm{kHz}$ is the revolution frequency, $E_{0}=8.938 \mathrm{GeV}$ is the nominal beam energy, $\eta=-8.9150 \times 10^{-3}$ is the slip factor, and $\beta$ is the nominal velocity of the beam particles with respect to the velocity of light. When the bunch is small or when the rf is exactly linear, the above expressions reduce to

$$
\widehat{\Delta E}=\frac{\beta^{2} E_{0}}{|\eta|} \omega_{s} \hat{\tau}, \quad A=\sqrt{\frac{\pi e V \beta^{2} E_{0} h}{2|\eta|}} \omega_{0} \hat{\tau}^{2}, \quad \omega_{s}=\omega_{0} \sqrt{\frac{|\eta| h e V}{2 \pi \beta^{2} E_{0}}}
$$

where $\omega_{s} / 2 \pi$ is small-amplitude the synchrotron frequency.

The fractional energy spread is $1.506 \times 10^{-3}$, which is within the energy aperture of the Recycler of $0.25 \%$, the total captured bunch length of 100 ns is just what the $g-2$ experiment originally sought for, and the $V=54.26 \mathrm{kV}$ is well below the maximum attainable $65 \mathrm{kV}$ of the Main Injector 2.5-MHz rf system.* Unfortunately, adiabatic debunching and adiabatic capture can never be ideal. There will be an increase in longitudinal emittance in each step of the beam maneuvering. The shorter the debunching and capture times, the larger the increase in beam emittance. At $2.5-\mathrm{MHz}$ rf voltage $65 \mathrm{kV}$, a bunch of half width $60 \mathrm{~ns}$ has a bunch area of $3.26 \mathrm{eVs}$, according to Eq. (2.2). If the initial Booster bunches are each of area $0.1 \mathrm{eVs}$, the present $2.5-\mathrm{MHz}$ rf system can allow the super bunch area to increase by $3.26 /(21 \times 0.1)-1=55 \%$. The amount of allowable emittance increase will be reduced, however, if the emittance of each incoming Booster bunch is larger than 0.1 eVs. As will be shown below, because of the time limitation for the beam preparation, both the debunching and re-capture cannot be highly adiabatic and large increase in emittance will be encountered. To ensure the final super bunches to have a full width of $120 \mathrm{~ns}$, an upgrade of the $2.5-\mathrm{MHz}$ rf voltage will be necessary. In short, more practical beam maneuvering should be carefully investigated. In below, we will study these non-ideal problems one by one.

*At this moment, the 2.5-MHz rf at the Main Injector allows a maximum of $65 \mathrm{kV}$ in operation, although the rf system can tolerate up to $75 \mathrm{kV}$. 


\section{Realistic Adiabatic Debunching}

Adiabatic debunching is accomplished by lowering the rf voltage adiabatically from $V=V_{i}$, the voltage matched to the original Booster bunch, to a voltage $V=V_{f}$, at a rate so slow that the relative change in bucket height or bucket area $A_{\text {buc }}$ at any instant is very much slower than the instantaneous synchrotron frequency $\omega_{s}$. Mathematically, this can be expressed as [1]

$$
\omega_{s}=-\frac{n_{a d}}{A_{\mathrm{buc}}} \frac{d A_{\mathrm{buc}}}{d t},
$$

where the adiabatic parameter $n_{a d}$ regulates the degree of adiabaticity and should therefore be a big number. Since both the bucket area $A_{\text {buc }}$ and the synchrotron frequency $\omega_{s}$ are proportional to $\sqrt{V}$, this translates into

$$
\frac{\omega_{s}}{\sqrt{V}}=-\frac{n_{a d}}{2 V^{3 / 2}} \frac{d V}{d t}
$$

where the left side $\omega_{s} / \sqrt{V}$ is time-independent. Solution of this equation is

$$
V(t)=\frac{V_{i}}{\left(1+\omega_{s i} t / n_{a d}\right)^{2}},
$$

where $\omega_{s i}$ is the synchrotron frequency at start or $t=0$, when the rf voltage $V_{i}$. Thus perfect adiabaticity implies debunching duration $t_{d}=\infty$ when the rf voltage is reduced to $V=0$. In practice, the debunching duration $t_{d}$ is finite and the rf voltage is reduced to

$$
V_{f}=\frac{V_{i}}{\left(1+\omega_{s i} t_{d} / n_{a d}\right)^{2}} .
$$

The adiabatic parameter $n_{a d}$ in above can be eliminated and the debunching rf voltage becomes

$$
V(t)=\frac{V_{i}}{\left[1+\left(\sqrt{\frac{V_{i}}{V_{f}}}-1\right) \frac{t}{t_{d}}\right]^{2}} .
$$

This is the voltage program of adiabatic debunching in the Main Injector which depends on the three input parameters $V_{i}, V_{f}$, and $t_{d}$. However, for a debunching that is adiabatic, the three entries must guarantee a large adiabatic parameter

$$
n_{a d}=\frac{\omega_{s i} t_{d}}{\sqrt{\frac{V_{i}}{V_{f}}}-1} .
$$


Let us estimate how much time can be allocated to adiabatic debunching and adiabatic capture into $2.5-\mathrm{MHz}$ buckets. At the end of the capture, the 2.5- $\mathrm{MHz}$ rf voltage will be at its maximum of $65 \mathrm{kV}$. The four super bunches will be transferred to the $g-2$ ring one by one. The time interval between successive transfer will be half a synchrotron period, which is $10.7 \mathrm{~ms}$. The total transfer duration for the four super bunches will be $32.2 \mathrm{~ms}$, which is one-and-a-half synchrotron periods. The total time for the beam manipulation must be completed within two Booster cycles or $133 \mathrm{~ms}$. In other words, there can be only $\sim 100 \mathrm{~ms}$ for both the adiabatic debunching and adiabatic capture. Of these two operations, adiabatic capture is more stringent, otherwise the bunch half length cannot be shortened to the required $120 \mathrm{~ns}$. In other words, the emittance increase is usually much larger during capture than debunching. The consensus is to allocate about $10 \mathrm{~ms}$ for adiabatic debunching and about $90 \mathrm{~ms}$ for adiabatic capture.

We would like to study the adiabatic debunching of a 53-MHz Booster bunch. We choose the half bunch length to be $\hat{\tau}=3 \mathrm{~ns}$. With a bunch area of $A=0.1 \mathrm{eVs}$, the half energy spread is $\widehat{\Delta E}=10.7 \mathrm{MeV}$. To maintain this bunch, the 53-MHz rf in the Main Injector should be at the voltage $V_{i}=470 \mathrm{kV}$. The adiabatic debunching will start from this initial voltage and decreases according to the voltage program of Eq. (3.6) or (3.8) to the final value of $V_{f}$. For ideal adiabatic debunching, the rf voltage reaches $16.0 \mathrm{kV}$ when the area 53-MHz rf bucket equals to the bunch area, and reaches $6.49 \mathrm{kV}$ when the $53-\mathrm{MHz} \mathrm{rf}$ bucket height equals to $A / 2 t_{b}=2.64 \mathrm{MeV}$, the half energy spread of the perfect adiabatically debunching beam, where $t_{b}=18.9 \mathrm{~ns}$ is the width of the 53-MHz rf bucket. As a result, the finally rf voltage must be chosen to be less than $6.49 \mathrm{kV}$. To ensure adiabaticity, the adiabatic parameter should be chosen as $n_{a d} \gtrsim 3$. Figure 1 shows the choice of debunching duration $t_{d}$ and final rf voltage $V_{f}$ for various adiabatic parameters. If we choose $n_{a d}=4$, we can let the final rf voltage be $V_{f}=4 \mathrm{kV}$. The debunching duration will be $t_{d}=10.5 \mathrm{~ms}$, around our allocation. Figure 2 shows that debunched beam in the longitudinal phase space at $n_{a d}=3.5,4.0$, and 4.5. The corresponding final rf voltages are, respectively, $V_{f}=3.40$, 4.34 , and $5.37 \mathrm{kV}$.

These simulations show that, first, debunching in roughly $10 \mathrm{~ms}$ is possible. Second, there is an increase in beam emittance. Instead of the ideal energy spread of $\widehat{\Delta E}=2.64 \mathrm{MeV}$, The spread appears to be slightly more than $\widehat{\Delta E}=3.0 \mathrm{MeV}$. If the injected Booster bunches have half width smaller than $3 \mathrm{~ns}$, the matching rf voltage will be larger than $V_{i}=470 \mathrm{kV}$. At the same adiabaticity, the debunching time will be longer correspondingly. The increase will be very minimal, however, because the rf voltage drops very fast at the beginning. As 


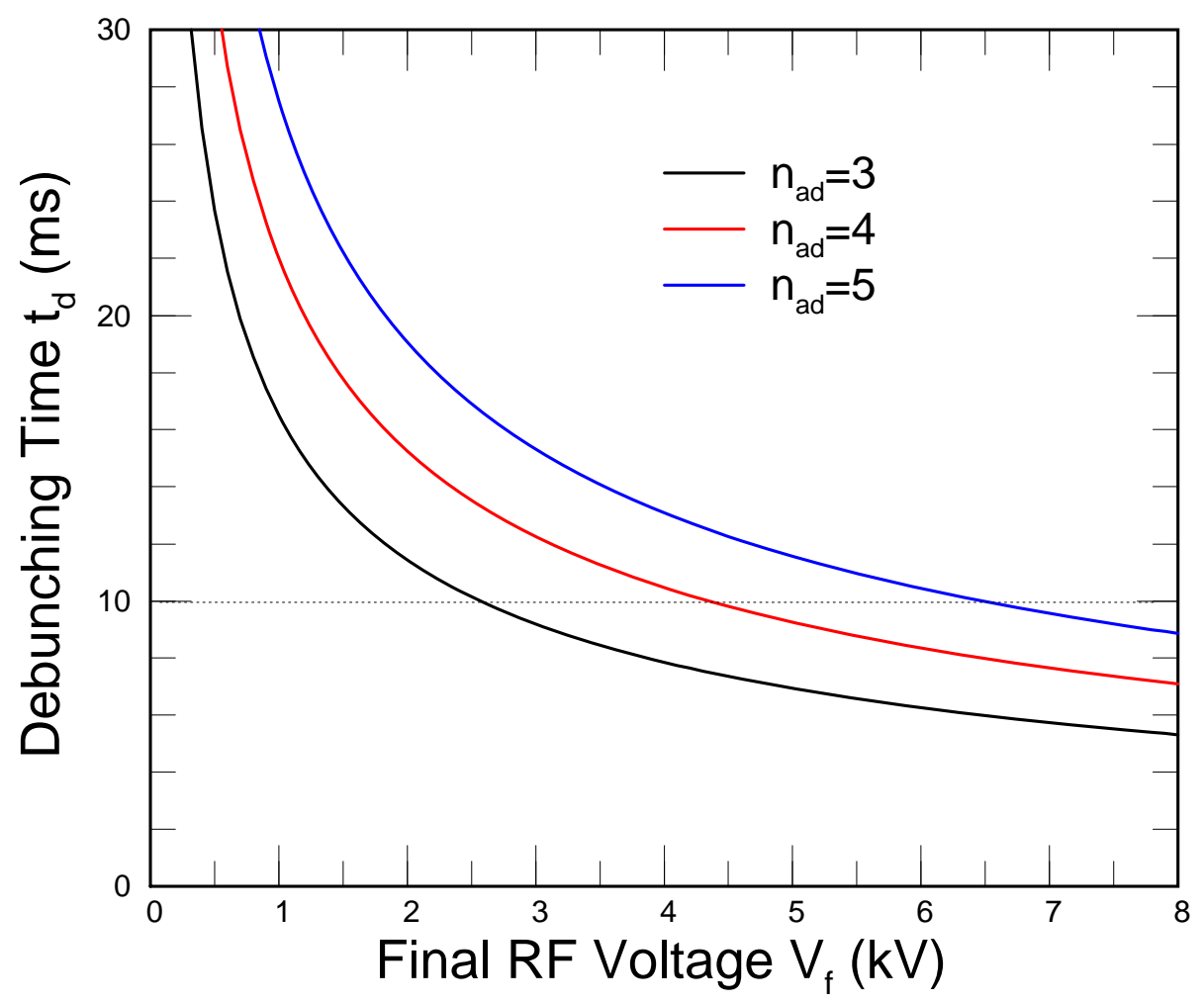

Figure 1: (Color) The choice of debunching duration $t_{d}$ and final rf voltage $V_{f}$ at various adiabatic parameters $n_{a d}$.

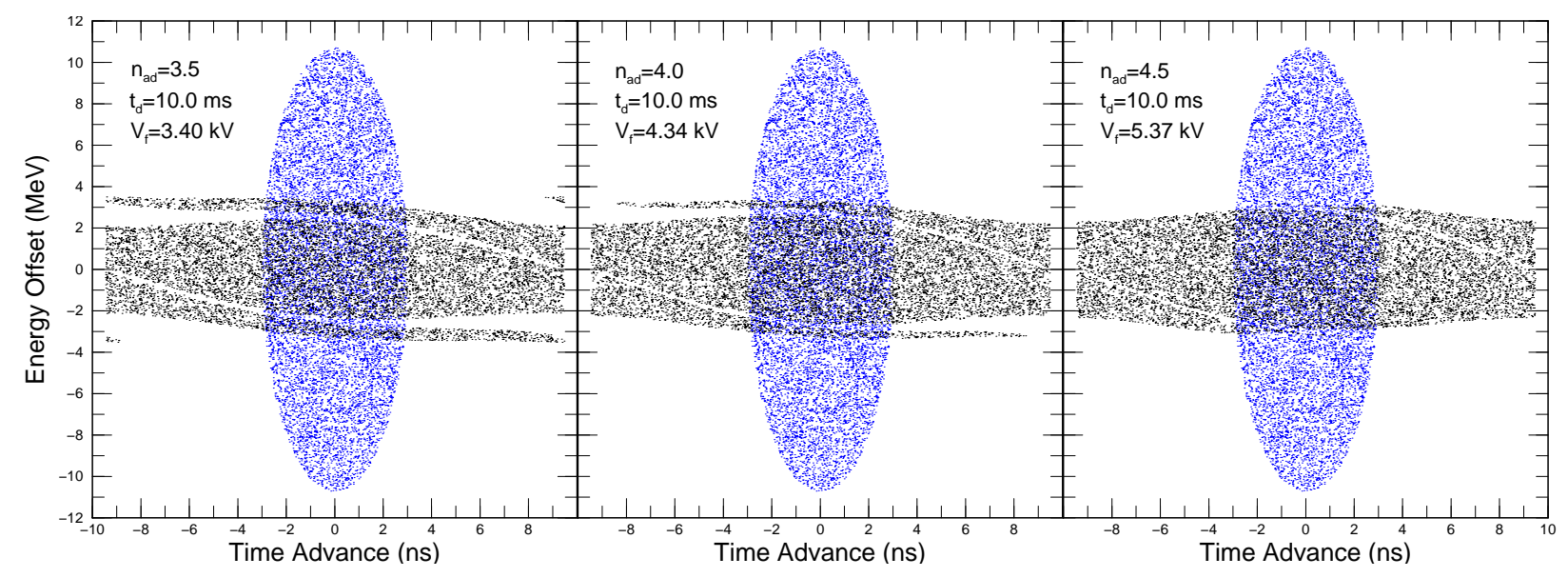

Figure 2: (Color) The initial $53-\mathrm{MHz}$ bunch of area $0.1 \mathrm{eVs}$ is shown as blue dots. The final distribution is shown in black. In total, then thousand macroparticles are used. The initial distribution is uniform. The three plots show debunching of duration $\sim 10 \mathrm{~ms}$ with adiabatic parameter $n_{a d}=3.5,4.0$, and 4.5, corresponding, respectively, to final rf voltage $V_{f}=3.40,4.34$, and $5.37 \mathrm{kV}$. 


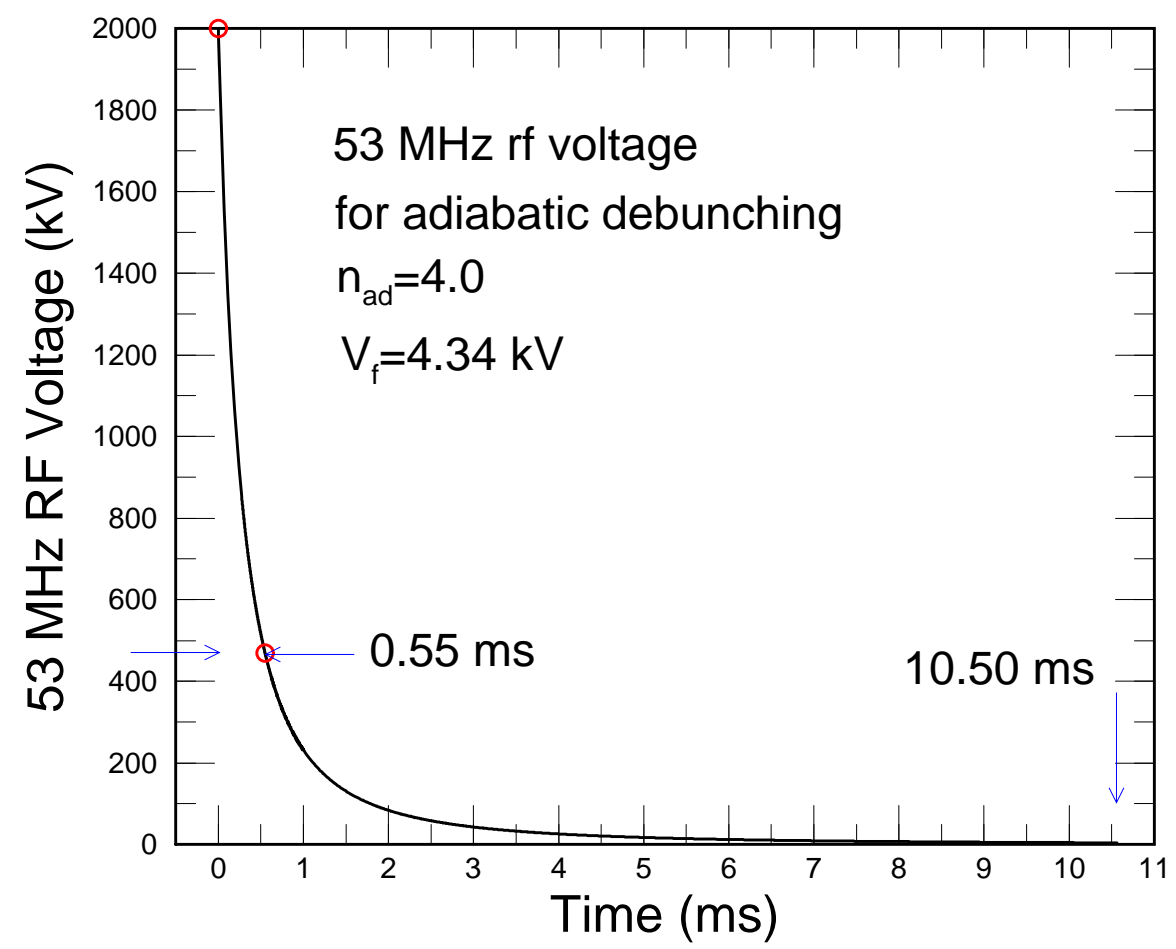

Figure 3: ((Color) Adiabatic debunching rf voltage program at the Main Injector for adiabaticity $n_{a d}=4.0$ and final rf voltage $V_{f}=4.34 \mathrm{kV}$. The plot show that if we start from $V_{i}=2 \mathrm{MV}$ instead of $407 \mathrm{kV}$, the debunching time is lengthened by $0.55 \mathrm{~ms}$ only.

an example, with adiabaticity $n_{a d}=4.0$ and final $\mathrm{rf}$ voltage $V_{f}=4.34 \mathrm{kV}$, the debunching rf voltage program in Fig. 3 shows that even if we start the debunching at Main Injector 53$\mathrm{MHz}$ rf voltage $V_{i}=2 \mathrm{MV}$ rather than $470 \mathrm{kV}$ as in the center plot of Fig. 2, the debunching duration will be longer by $0.55 \mathrm{~ms}$ only. which is too small to be an issue of concern.

It is possible that the area of each Booster bunch injected into the Main Injector is larger than $A=0.1 \mathrm{eV}$. We also perform simulations with the initial bunch area $10 \%$ larger $(A=0.11 \mathrm{eVs})$ at $n_{a d}=4$ and $V_{f}=4 \mathrm{kV}$. The energy spread of the debunched beam is found to be slightly larger than $3 \mathrm{MeV}$. Usually a bunch has much less particles at its edges. Thus the fraction of particles shoots over $\widehat{\Delta E}=3 \mathrm{MeV}$ should be small. This energy spread can be made smaller by allowing the adiabatic debunching duration to be made somewhat longer (by increasing $n_{a d}$ and/or decreasing $V_{f}$ ), because there can be an overlap of the adiabatic debunching process and the adiabatic capture process. For this reason, we will take $\widehat{\Delta E}=3 \mathrm{MeV}$ as the energy spread of the debunched beam in the study of adiabatic capture below. For 21 booster bunches destined to be coalesced into a super bunch, the debunched beam to be capture will now have a longitudinal area of $2.39 \mathrm{eVs}$. 


\section{Realistic Adiabatic Capture [2]}

The theory of adiabatic capture follows the same assumption of Eq. (3.4) as in adiabatic debunching. The only difference is that the negative sign on the right side is absent because the bucket area is now increasing. The voltage program is now

$$
V(t)=\frac{V_{i}}{\left(1-\omega_{s i} t / n_{a d}\right)^{2}}
$$

where $\omega_{s i} / 2 \pi$ is the synchrotron frequency at the start of the capture when the rf voltage is $V_{i}$. For a capture duration $t_{c}$, the final rf voltage is given by

$$
V_{f}=\frac{V_{i}}{\left(1-\omega_{s i} t_{c} / n_{a d}\right)^{2}}
$$

Eliminating the adiabatic parameter $n_{a d}$, the rf voltage program becomes

$$
V(t)=\frac{V_{i}}{\left[1-\left(1-\sqrt{\frac{V_{i}}{V_{f}}}\right) \frac{t}{t_{c}}\right]^{2}} .
$$

Again there are three parameters: the capture duration $t_{c}$ plus the initial and final rf voltages $V_{i}$ and $V_{f}$. However, they must guarantee a large adiabatic parameter

$$
n_{a d}=\frac{\omega_{s i} t_{c}}{1-\sqrt{\frac{V_{i}}{V_{f}}}}
$$

so that the process is adiabatic. It is obvious that the final $\mathrm{rf}$ voltage will be the maximum $65 \mathrm{kV}$ provided by the $2.5-\mathrm{MHz}$ rf system. This leaves us with only one parameter if we wish to set $n_{a d} \sim 4$, for example.

First let us determine what type of super bunch the 2.5-MHz rf can support. For a bunch of area $2.389 \mathrm{eVs}$ and half length $\hat{\tau}$, the required matching rf voltage can be computed, and is displayed in Fig. 4. At the highest possible voltage of $65 \mathrm{kV}$, a perfectly matched bunch of area $2.389 \mathrm{eVs}$ is $50.97 \mathrm{~ns}$. In other words, even perfectly ideal adiabatic capture will produce a 2.5-MHz bunch that has full length slightly longer than $100 \mathrm{~ns}$, the bunch length the $g-2$ project originally sought for. On the other hand, with the revised larger allowable bunch length of $120 \mathrm{~ns}$, only $34.78 \mathrm{kV}$ is needed if the capture is perfectly ideal. Keeping the half bunch length fixed at $60 \mathrm{~ns}$, the maximum $65 \mathrm{kV}$ rf voltage can match the super bunch 


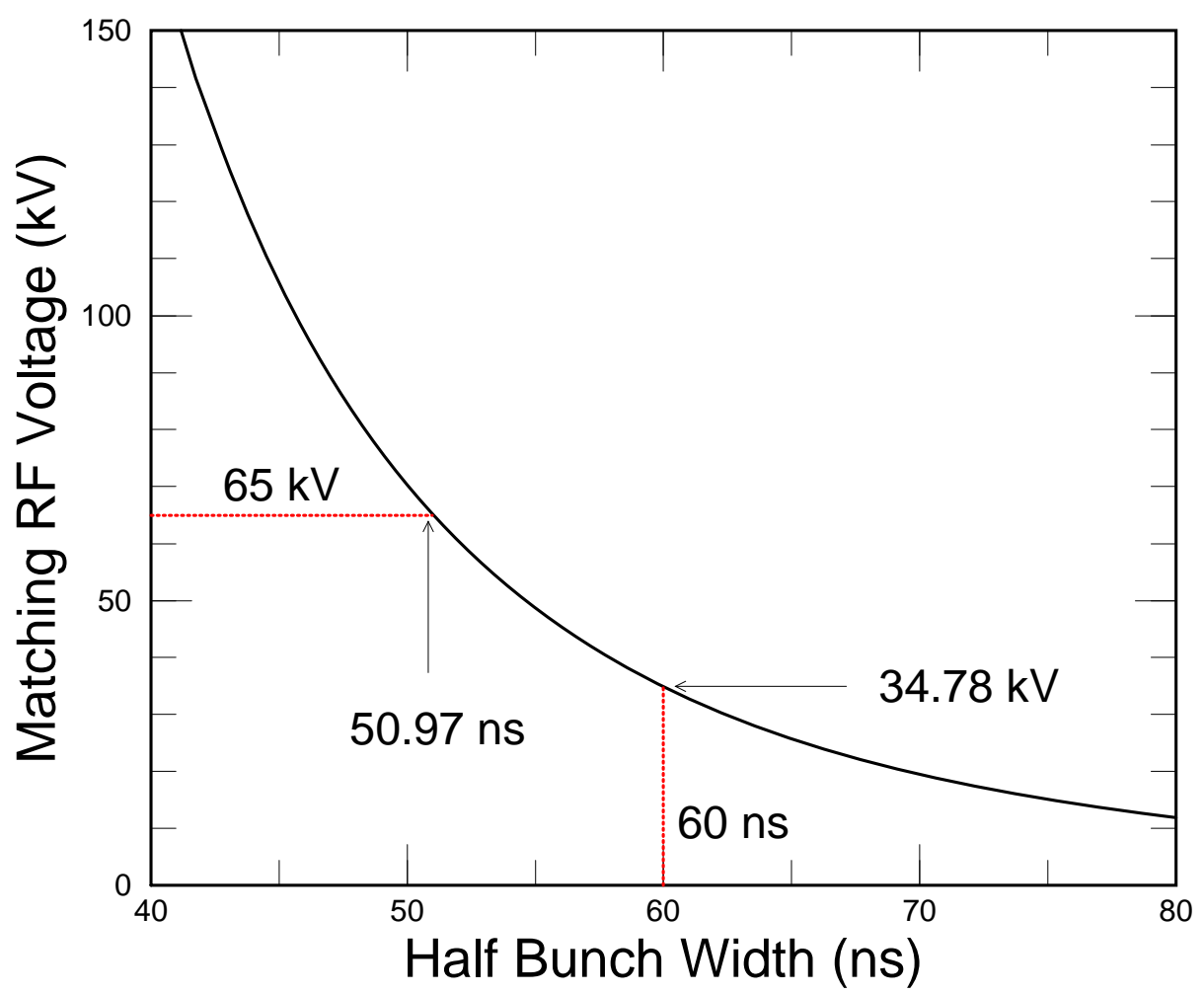

Figure 4: (Color) 2.5-MHz rf voltage required to support a bunch of area $2.389 \mathrm{eVs}$ versus the half bunch length.

of area $\sqrt{65 / 34.78}=1.367$ larger (see Eq. (2.1) or (2.3). As a result, it is very plausible that we can capture the beam into a bunch less than $120 \mathrm{~ns}$ long with the present $65 \mathrm{kV} 2.5 \mathrm{MHz}$ rf, allowing an increase in bunch area of $36.7 \%$ during the capture.

Next question of concern is the choice of initial rf voltage $V_{i}$. Some guidance can be obtained as below. The rf voltage required to setup a $2.5-\mathrm{MHz}$ bucket of the same area of the super bunch before capture is $0.985 \mathrm{kV}$. The rf voltage required to setup a $2.5-\mathrm{MHz}$ bucket having a bucket height equal to the maximum energy spread of the beam, $3 \mathrm{MeV}$, before capture is $0.399 \mathrm{kV}$. Thus the choice of initial rf voltage $V_{i}$ should be around $0.399 \mathrm{kV}$. Figure 5 shows the capture time as a function of the initial rf voltage for various adiabatic parameters $n_{a d}$. Since the capture time is limited to roughly $t_{c}=90 \mathrm{~ms}$, the choice of capture parameters appears to be around $n_{a d}=2.0$ and $V_{i}=0.30 \mathrm{kV}$ or $n_{a d}=3$ and $V_{i}=0.63 \mathrm{kV}$. These are shown in the first two plots of Fig. 6. We see that most the super bunch is between $\tau= \pm 50 \mathrm{~ns}$, but a small amount from the tails extends over more than $\tau= \pm 60 \mathrm{~ns}$. In any case, it appears that $95 \%$ of the captured bunch is inside $\tau= \pm 60$ ns. The third plot shows a capture with $n_{a d}=5.0$ and $V_{i}=1.05 \mathrm{kV}$, and appears to be wider than the first two 


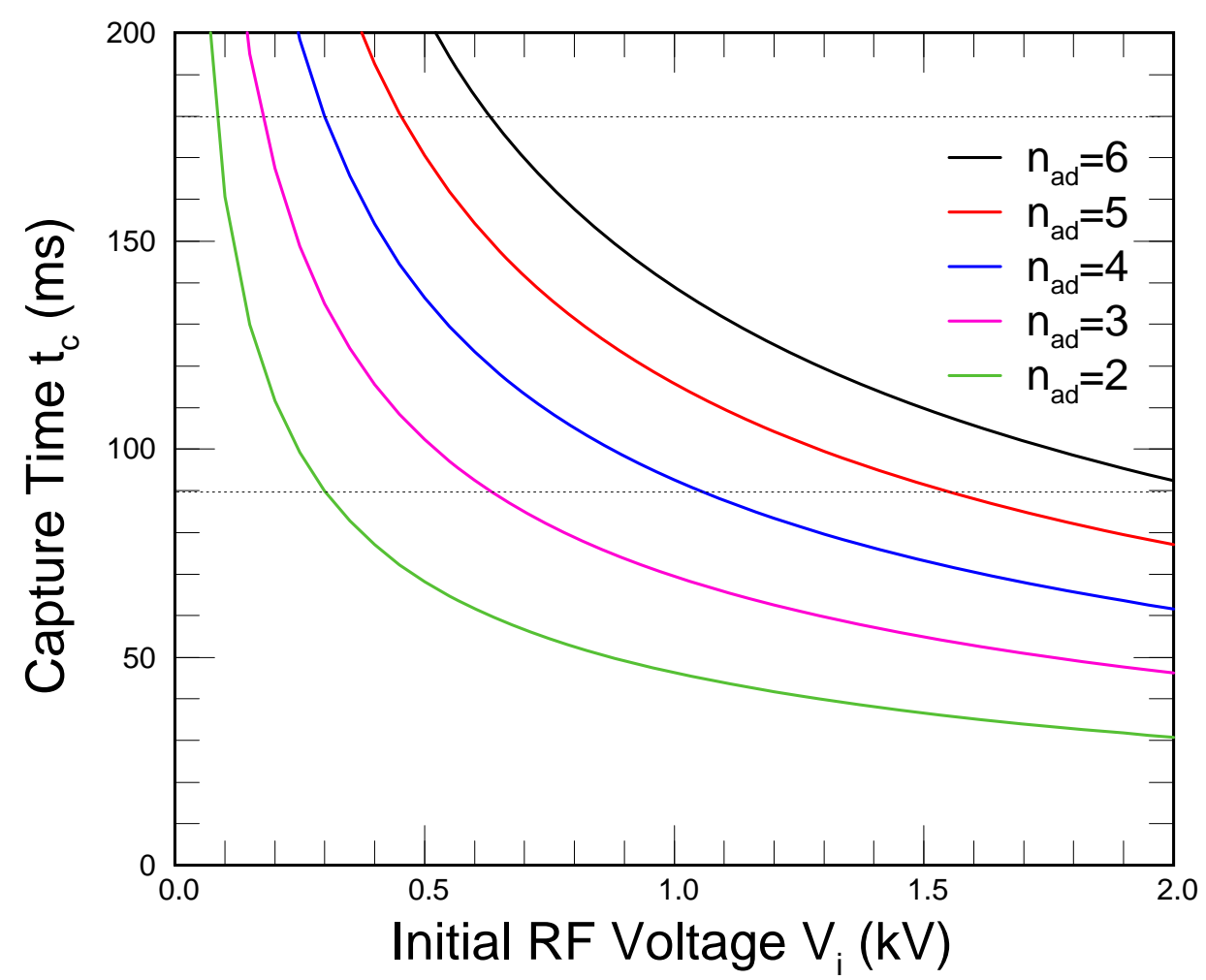

Figure 5: (Color) The choice of capture duration $t_{c}$ and initial $\mathrm{rf}$ voltage $V_{i}$ at various adiabatic parameters $n_{a d}$.

captures, although $95 \%$ of the bunch is still within $\tau= \pm 60$ ns. The implication is that a lower initial rf voltage can be more important than higher degree of adiabaticity. The initial rf voltage should produce a bucket height smaller than, or at least not exceeding by much, the initial spread of the debunched beam.

Besides the baseline scheme, there is an alternate scheme that can allow the capture time to be as long as $t_{c}=180 \mathrm{~ms}$. The simulations of such a scenario are shown in Fig. 7, for adiabatic parameter $n_{a d}=4.0,5.0$, and 6.0. The corresponding initial rf voltages are, respectively, $V_{i}=0.30,0.45$, and $0.63 \mathrm{kV}$. For all the three cases, $95 \%$ of the beam particles are certainly contained inside $\tau= \pm 60 \mathrm{~ns}$ and may be within $\pm 50 \mathrm{~ns}$ as well. Among them, the case of $n_{a d}=5.0$ corresponding to $V_{i}=0.45$ appears to be the best. In general, the bunch shape is much better than when the allowed capture duration is $t_{c}=90 \mathrm{~ms}$. Obviously, this is the result of allowing higher degree of adiabaticity. 


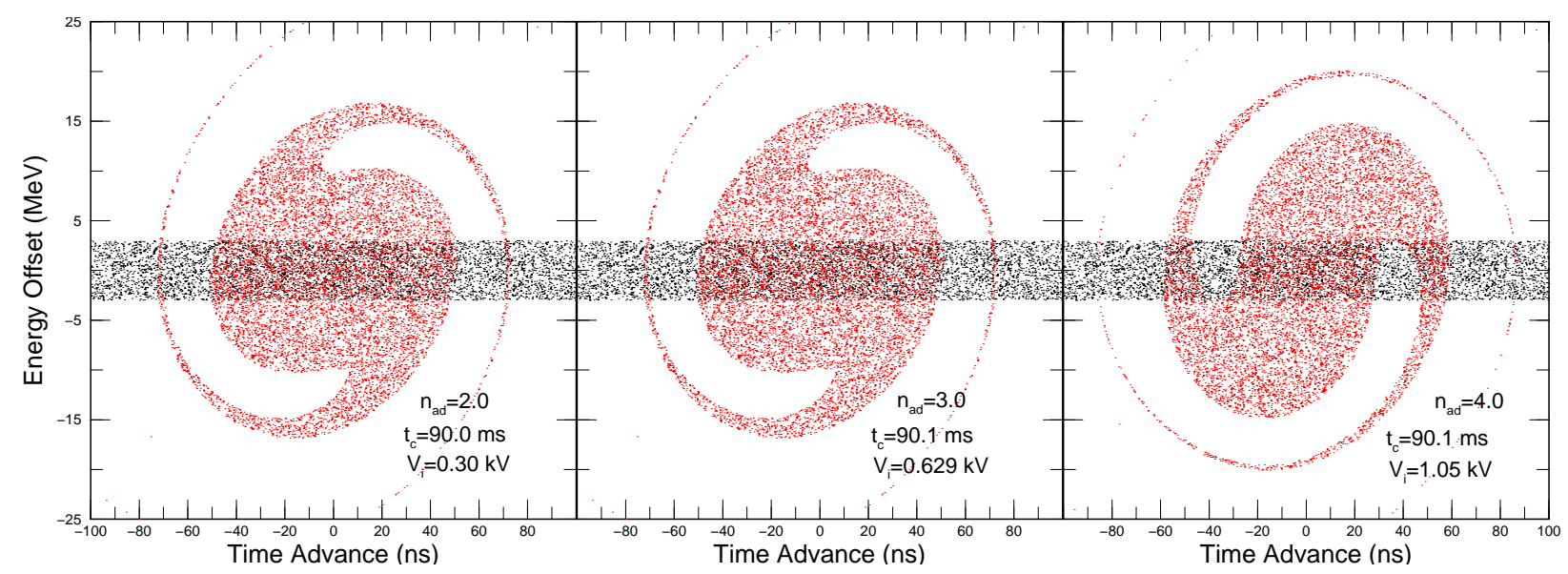

Figure 6: (Color) The initial totally debunched beam, shown as black dots, is of half energy spread $\widehat{\Delta E}=3 \mathrm{MeV}$ and half width $\hat{\tau}= \pm 198.8 \mathrm{~ns}$ (not all of it is shown). It is captured to become a $2.5-\mathrm{MHz}$ super bunch from rf voltage $V_{i}$ to $V_{f}=65 \mathrm{kV}$. The capture time is approximately $t_{c}=90 \mathrm{~ms}$. Final longitudinal distribution is shown as red dots. Adiabatic parameter $n_{a d}=2,3$, and 4 are used in the three plots, corresponding, respectively, to initial $\mathrm{rf}$ voltage $V_{i}=0.30,0.629$, and $1.05 \mathrm{kV}$. Ten thousand macroparticles are employed in the each simulation.

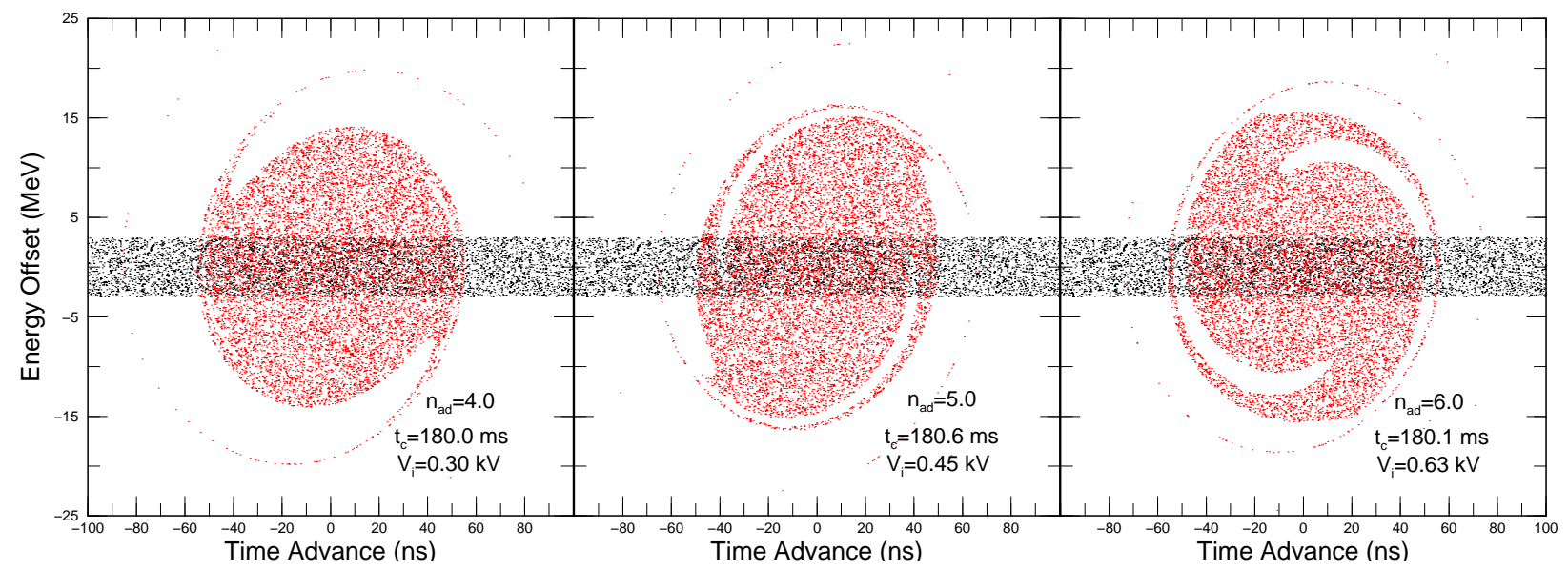

Figure 7: (Color) The initial totally debunched beam, shown as black dots, is of half energy spread $\widehat{\Delta E}=3 \mathrm{MeV}$ and half width $\hat{\tau}= \pm 198.8 \mathrm{~ns}$ (not all of it is shown). It is captured to become a $2.5-\mathrm{MHz}$ supper bunch from rf voltage $V_{i}$ to $V_{f}=65 \mathrm{kV}$. The capture time is approximately $t_{c}=180 \mathrm{~ms}$. Final longitudinal distribution is shown as red dots. Adiabatic parameter $n_{a d}=4,5$, and 6 are used in the three plots, corresponding, respectively, to initial rf voltage $V_{i}=0.30,0.45$, and $0.65 \mathrm{kV}$. Ten thousand macroparticles are employed in each simulation. 


\section{$5 \quad$ Future 2.5-MHz RF System in the Recycler}

The present 2.5-MHz rf system in the Main Injector will be moved to the Recycler and be improved to accommodate the creation of the super proton bunches for the $g-2$ project. Here, we wish to estimate the highest rf voltage that should be installed [3].

The momentum aperture of the Recycler is $0.25 \%$, or the energy offset of $\widehat{\Delta E}=$ 22.35 MeV. The rf voltage $V$ required to set up a bunch of this energy offset and half bunch length $\hat{\tau}=60$ ns is given by Eq. (2.1), which turns out to be $V=106.3 \mathrm{eV}$. This implies that when half bunch length of $\hat{\tau}=60 \mathrm{~ns}$ is required, there is no reason to upgrade the rf voltage to more than $106.3 \mathrm{kV}$. At that voltage, the matched bunch area is, according to Eq. (2.2), $A=4.17 \mathrm{eVs}$. If we take the same beam area as $2.39 \mathrm{eVs}$ before capture, we can allow a maximum emittance increase of $\sqrt{4.17 / 2.39}=74.5 \%$ during capture. This gives us the guidance that in the present machine studies at the Main Injector, we must limit the emittance growth during adiabatic capture to $\lesssim 74.5 \%$. Otherwise, our expectation of the beam preparation will fail. We learn from above that without emittance growth, the half bunch length obtainable from a $65-\mathrm{kV}$ rf is $50.97 \mathrm{~ns}$ (Fig. 5). This implies that we must try at this moment produce a $2.5 \mathrm{MHz}$ bunch of half width $\leq 50.97 \times \sqrt{1.745}=67.3 \mathrm{~ns}$. Otherwise, in the future even when the $2.5-\mathrm{Hz}$ rf voltage is upgraded, it will not be able to produced super bunches of half length $\hat{\tau}=60$ ns.

Alternately, if super bunches of half length $\hat{\tau}=50 \mathrm{~ns}$ is required, the maximum bunch area can be set up at $\widehat{\Delta E}=22.35 \mathrm{MeV}$ is $A=3.49 \mathrm{eVs}$, or the allowable emittance increase is reduced to $45.9 \%$ during capture. The rf voltage required for such a bunch will be $V=150 \mathrm{kV}$.

With a higher rf final voltage, the capture time will be increased correspondingly. However, this increase will be minimal, because the voltage problem of the capture process increases very rapidly near the end of the capture. Figure 8 shows the rf voltage program at $n_{a d}=3.0$ and $V_{i}=0.629 \mathrm{kV}$. When the final $\mathrm{rf}$ voltage is raised to $V_{f}=100 \mathrm{kV}$ instead of $65 \mathrm{kV}$, the capture time is lengthened by $1.91 \mathrm{~ms}$ only.

Simulations tell us that a higher rf voltage can produce a smoother captured bunch if larger capture time is allowed. We wish to point out, however, that a higher rf voltage is inefficient in producing a narrower bunch. This is obvious if we look into Eq. (2.3). At fixed bunch area, $V \propto \hat{\tau}^{4}$. For example, if we wish to further shorten the bunch by $5 \%$, we need an rf voltage which is $\sim 22 \%$ larger. 


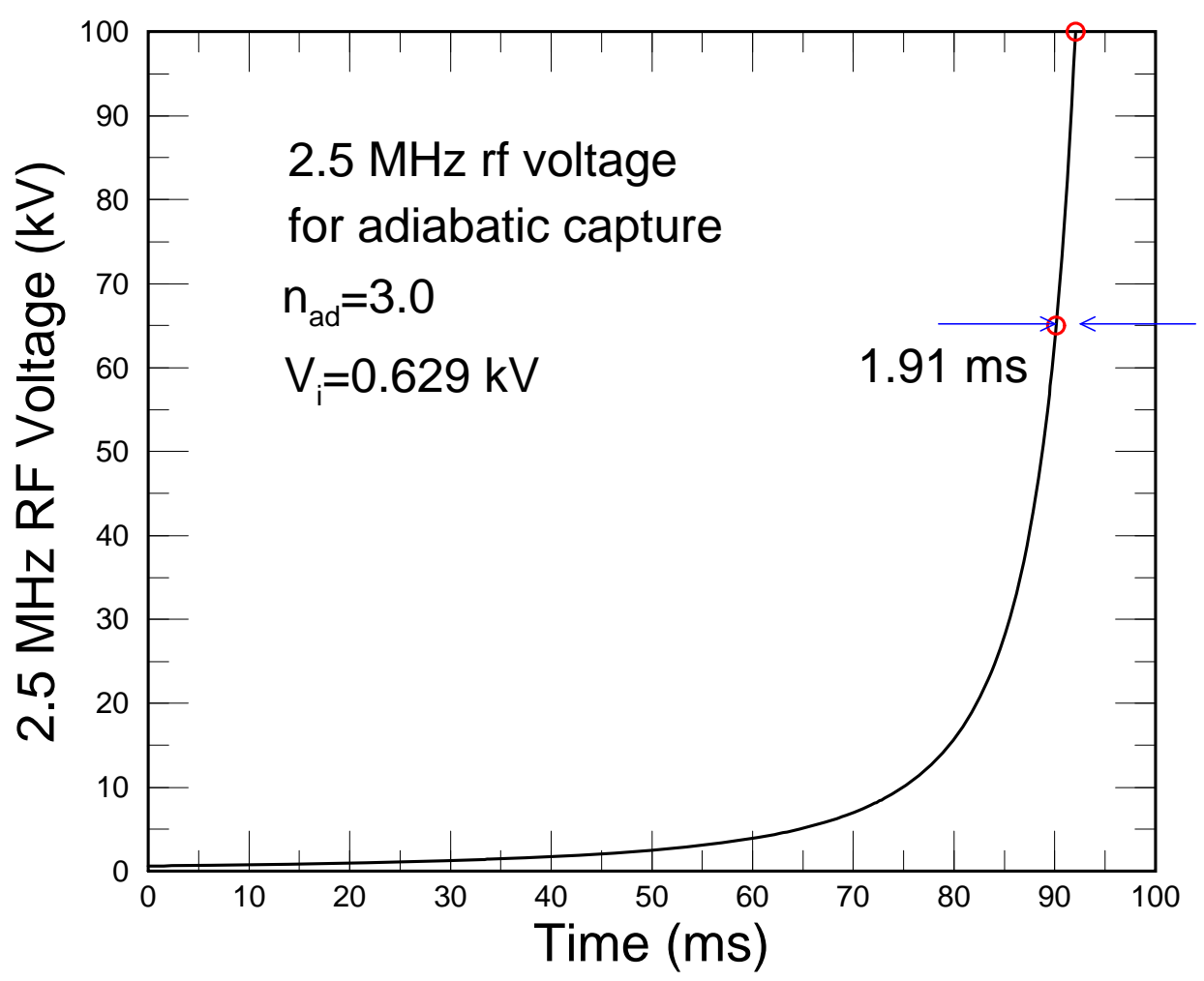

Figure 8: (Color) Adiabatic capture rf voltage program at the Main Injector for adiabaticity $n_{a d}=3.0$ and initial $\mathrm{rf}$ voltage $V_{i}=0.629 \mathrm{kV}$. The plot show that if we raise the final $\mathrm{rf}$ voltage to $V_{f}=100 \mathrm{kV}$ instead of $65 \mathrm{kV}$, the debunching time is lengthened by $1.91 \mathrm{~ms}$ only.

\section{Particle Leakage}

During adiabatic debunching or capture, particles move from one bucket to the neighboring buckets. This is illustrated in Fig. 2. The top and bottom triangular pieces of the debunching beam in each plot are formed by particles drifting from the adjacent $53-\mathrm{MHz}$ buckets. Since we are debunching only one Booster batch in the Main Injector, particles from bunches at the two ends of the batch will drift away from the batch. This leads to particle loss and left-behind dc current, both of which are undesirable.

One method to alleviate particle leakage is to place rf barriers on both sides of the batch. The integrated barrier intensity to hold a beam of maximum energy spread $\widehat{\triangle E}= \pm 3 \mathrm{MeV}$ is $[4]$

$$
\left[V_{b} T_{b}\right] \equiv \int V_{b}(t) d t=\frac{|\eta| T_{0}(\widehat{\Delta E})^{2}}{2 \beta^{2} E_{0}}=51 \mathrm{kV} \text {-ns. }
$$

The present available barrier rf has a peak voltage of $V_{b}=8 \mathrm{kV}$, meaning that the penetration 


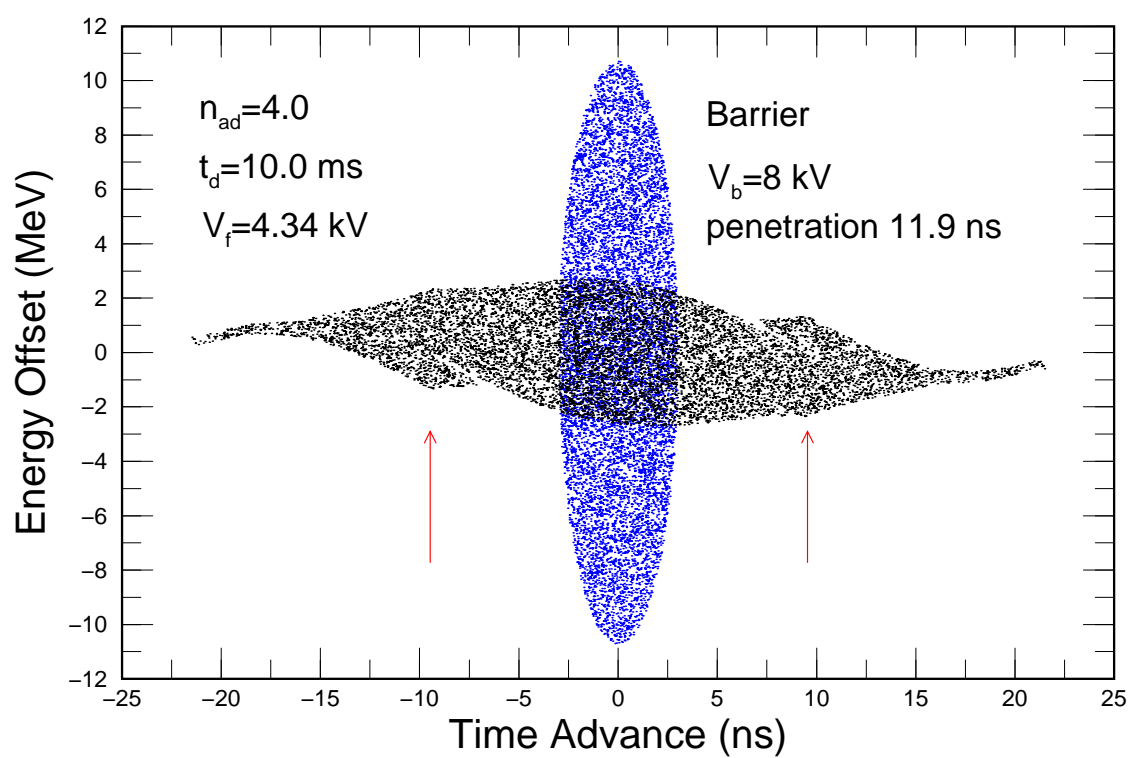

Figure 9: (Color) Same as center plot of Fig. 2, but with barrier voltages $V_{b}= \pm 8 \mathrm{kV}$ on the right and left of the 53-MHz bucket. The penetration into neighboring buckets has been $11.9 \mathrm{~ns}$, which is large. The edges of the $53-\mathrm{MHz}$ bucket are marked by red arrows.

into the barrier will be $\tau_{\text {leak }}=6.3 \mathrm{~ns}$, if a square barrier pulse is assumed. Unfortunately, this penetration accounts for $2 / 3$ the half bucket width $t_{b} / 2=9.47 \mathrm{~ns}$. The implication is that the $53-\mathrm{MHz}$ rf voltage wave increases from the edge of the bucket and counteract the barrier voltage. Thus more integrated barrier strength will be required to block the beam particles from leakage. Simulation result in Fig. 9 shows that at $V_{b}=8 \mathrm{kV}$, the penetration increases to $11.9 \mathrm{~ns}$, which is more than the half width of the 53- $\mathrm{MHz}$ bucket. The only way to reduce the effect of the $53-\mathrm{MHz}$ rf voltage wave at the neighboring buckets is to reduce the penetration into neighboring buckets. To accomplish this, the barrier voltage must be increased. For example, if the barrier voltage can be doubled to $V_{b}=16 \mathrm{kV}$, the penetration will be reduced to 4.2 ns. At the same time, the shape of the debunched beam will be much smoother than what is depicted in Fig. 9. We wish to point out that in the debunching of the whole Booster batch, the barriers are placed only on the two ends of the batch. However, in the simulation depicted in Fig. 9, the barrier pair is placed on the two ends of one Booster bunch. We do this to simplify the simulation of the whole batch of 21 bunches to the simulation of only one bunch. Nevertheless, the effect of the barriers is well demonstrated.

During capturing, the same barrier voltage of $V_{b}=8 \mathrm{kV}$ will stop all leakage and result in a penetration of $6.3 \mathrm{~ns}$ into the adjacent $2.5-\mathrm{MHz}$ rf buckets. Unlike the debunching 


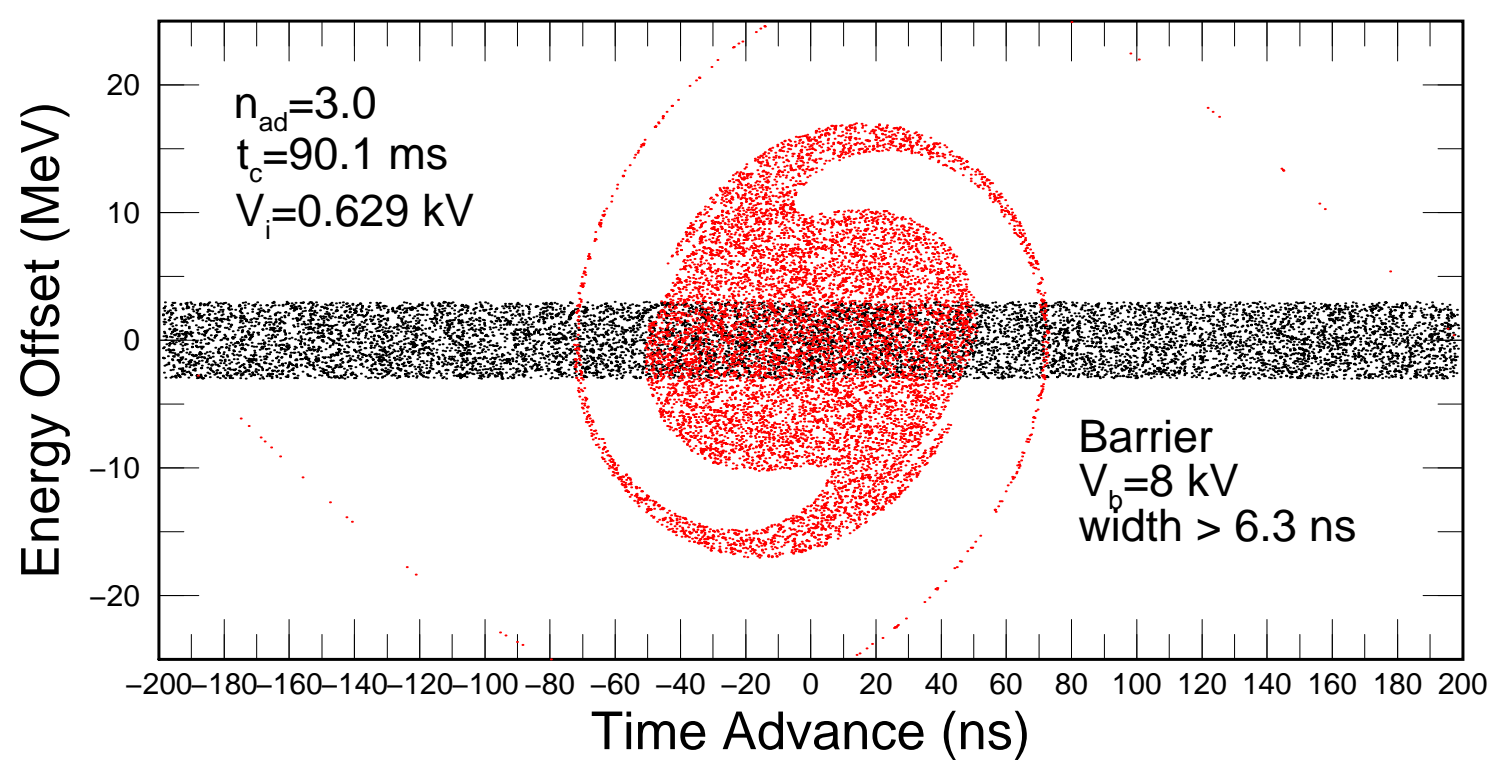

Figure 10: (Color) Same as center plot of Fig. 6, but with barrier voltages $V_{b}= \pm 8 \mathrm{kV}$ on the right and left of the 2.5-MHz bucket. Unlike Fig. 6, the whole 2.5-MHz bucket width of 398 ns is shown. No penetration into neighboring buckets is seen in the final frame of the capture. Earlier penetration in the capture process is $6.3 \mathrm{~ns}$.

process, this penetration is very small compared with the half width $t_{b} \sim 200 \mathrm{~ns}$ of the 2.5$\mathrm{MHz}$ rf bucket. The influence of the $2.5 \mathrm{-MHz}$ rf voltage wave is therefore very minimal. For this reason, the required integrated strength of the barrier to block particle leakage is truly $\left[V_{b} T_{b}\right]=51 \mathrm{kV}$-ns, as predicted by Eq. (6.14). Figure 10 shows a simulation of the capture with barrier voltages of $V_{b}=8 \mathrm{kV}$ on both side of the 2.5-MHz rf bucket. The adiabaticity and initial rf voltage are the same as in the middle plot of Fig. 6 . At the beginning of the capture, beam particles do leak into neighboring buckets, but are blocked by the barrier voltages $V_{b}= \pm 8 \mathrm{kV}$ after a penetration of $T_{b}=6.3 \mathrm{~ns}$. The beam distribution shown in red at the end of the capture is very similar to that in the middle plot of Fig. 6.

\section{Conclusion}

We have studied the adiabatic debunching of a Booster batch in the Main Injector and subsequent adiabatic re-capture into four super bunches, each of full width less than $120 \mathrm{~ns}$, if the tails of the bunches are not included. The time duration of the debunching is $\sim 10 \mathrm{~ms}$, while that of the re-capture is $\sim 90 \mathrm{~ms}$. The maximum voltage of the $2.5-\mathrm{MHz} \mathrm{rf}$ is $65 \mathrm{kV}$. 
The half energy spread is $\sim 15 \mathrm{MeV}$ or $\sim 0.17 \%$, well within the momentum aperture of $2.5 \%$ of the Main Injector.

The leakage of particles at the two ends of the Booster batch can be stopped by placing rf barriers at the two ends. Since the half width of the $53-\mathrm{MHz}$ bucket is $9.5 \mathrm{~ns}$, the barrier voltages can be strongly influenced by the $53-\mathrm{MHz}$ rf voltage wave during debunching. As a result, barrier penetration must be limited to less then $9.5 \mathrm{~ns}$, half width of the $53-\mathrm{MHz}$ bucket. We show that a barrier voltage of $8 \mathrm{kV}$ is not large enough for the job. If it is doubled to $16 \mathrm{kV}$, the barrier penetration can be reduced to $4.2 \mathrm{~ns}$. On the other hand, the influence of the $2.5-\mathrm{MHz}$ rf voltage to the barrier is very minimal during adiabatic capture, because of the very much larger width of the $2.5-\mathrm{MHz}$ bucket. A barrier voltage of $8 \mathrm{kV}$ will be large enough to stop all leakages.

\section{References}

[1] K.Y. Ng, Continuous Multiple Injections at the Main Injector, Phys. Rev. ST Accel. Beams 5, 061002 (2002), Fermilab Report FN-0715, 2002; K. Woodbury and K. Meisner, Main Injector Adiabatic Counter Phasing Specification Version 2.0, September 1999, unpublished; K.Y. Ng, Adiabatic Capture and Debunching, Fermilab Report FN-0943APC, 2012.

[2] K.Y. Ng, Theoretical Study of Proton Beam Preparation for the $g-2$ Experiment, Fermilab Report FN-0934-APC, 2011.

[3] K.Y. Ng, Some Issues on g-2 Proton Beam Preparation, Fermilab Report TM-2537APC, 2012.

[4] S.Y. Lee and K.Y. Ng, Particle Dynamics in Storage Rings with Barrier rf systems, Phys. Rev. E55, 5992 (1997). 